\title{
Impact of the light coupling on the sensing properties of photonic crystal cavity modes
}

\author{
Kumar Saurav*a,b, Nicolas Le Thomas ${ }^{\mathrm{a}, \mathrm{b}}$, \\ ${ }^{a}$ Photonics Research Group, Ghent University-imec, Technologiepark-Zwijnaarde 15, 9052 Ghent, \\ Belgium; ${ }^{\mathrm{b}}$ Center for Nano- and Biophotonics, Ghent University, 9000 Ghent, Belgium
}

\begin{abstract}
Optimally coupling light in an integrated Photonic crystal $(\mathrm{PhC})$ cavity is challenging, but crucial for improving their sensing properties. Here we experimentally investigate the impact of side coupling and in-line coupling on the transmission properties of integrated silicon $\mathrm{PhC}$ based air-slot cavities by probing the near field of the cavity mode with a nano fiber tip. These cavities were fabricated with standard deep UV lithography. Positioning this nano-tip near and inside $130 \mathrm{~nm}$ wide $\mathrm{PhC}$ slot cavity modifies the dielectric map of the cavity which perturbs the intensity scattered from the cavity surface. We show that the mapping of the nano tip induced intensity variations provides some insight about the nature of the confinement of electric field of the various modes of slot cavities. Such intensity maps carry moreover information about the cavity light coupling, which is useful for maximizing the intensity of PhC slot cavity modes.
\end{abstract}

Keywords: Photonic crystal cavities, side coupling, in-line coupling, Silicon nanophotonics.

\section{INTRODUCTION}

Photonic crystals (PhC) based cavities allow for both high quality (Q) factor and strong field confinement [1,2], which results in enhanced light-matter interaction within an extremely small volume. These unique properties are important for various applications such as cavity quantum electrodynamics, nonlinear optics, and optical sensing. In particular PhC cavities fabricated in silicon $(\mathrm{Si})$ makes them a promising candidate for integrated photonic biosensors, owing to their compatibility with Complementary Metal-Oxide-Semiconductor (CMOS) fabrication technology. These devices are increasingly being investigated for lab-on-a-chip applications due to their benefits such as biocompatibility, passive behavior and lack of the need for fluorescent labels [1-4]. The small footprint of this device, as small as a few microns, also presents opportunities for dense arrays of sensing elements in lab-on-a-chip applications.

Among various $\mathrm{PhC}$ cavities, air-slotted cavity has become a very interesting new player in the field of photonic biosensors which squeezes light down into small volume of air. This strong confinement of light in air is the key advantage of this architecture, as it promotes strong light-matter interactions with a substance of interest. We use a scattering near field optical technique to experimentally determine the sensing properties of such slotted $\mathrm{PhC}$ cavity mode. This technique makes use of a silica nano-tip fiber that moves in the near field of the $\mathrm{PhC}$ cavity and of a high numerical microscope objective that collects the field radiated out-of plane from the cavity. Measuring the intensity fluctuations of the cavity field that are induced by the nano tip via local perturbation of the refractive index allows us in principle to map the intensity profile of the cavity mode.

Here, we show that the input light coupling into the cavity mode can impact the electric field map of the cavity mode in an air-slot cavity. This study provides an inside about sensing properties of the cavity mode with which an analyte of interest will interact. In this article, we compare transmission properties of an in-line and side coupled air-slot cavities. From this study, we point out that there is a need for a better design of air-slot cavities in which mode excitation can be done more efficiently without making use a slot waveguide in the $\mathrm{PhC}$. 


\section{DESIGN AND FABRICATION OF PHOTONIC CRYSTAL SLOT CAVITIES}

In a typical PhC cavities, light-matter interaction occurs in the high-index material which only utilizes the evanescent tail of the confined optical mode for analyte detection and hence limits their sensitivity. This drawback can be overcome with the use of an air-slot cavity, i.e., a PhC cavity with a nanometer-wide slot designed in the cavity defect region. High refractive index contrast boundaries can result in a large discontinuity in the normal component of the electric field inside the low index medium which permits cavity to confine TE-polarized modes in the slot. The strong confinement of light in the hollow core (air) of the cavity is the key advantage of such cavities, as it enhances the light-matter interactions with matter of interest. One such air-slot based photonic crystal cavities have been originally proposed in Ref. [5]. The localized slot guided mode is formed by introducing a non-terminated linear air slot in the center of the line defect photonic crystal cavities, which is realized by local width modulation of a line-defect width (shifting air holes away from waveguide) as shown in figure 1 . This permits the cavities to have an ultra-small mode volume.

We have fabricated such air-slot cavities were fabricated on a $220 \mathrm{~nm}$ thick silicon-on-insulator wafer with a $2 \mu \mathrm{m}$ buried oxide (BOX) layer by using $193 \mathrm{~nm}$ deep-ultraviolet (UV) lithography. The fabricated air-slot cavity has a lattice constant of $460 \mathrm{~nm}$, a hole radius of $140 \mathrm{~nm}$ and slot width of $120 \mathrm{~nm}$. The $2 \mu \mathrm{m}$ thick BOX layer below the PhC was completely removed by etching for $30 \mathrm{~min}$ in buffered hydrofluoric (BHF) acid to achieve a freestanding $\mathrm{PhC}$ membrane. In order to avoid stiction, the etched sample was dried using critical point dryer (CPD). Fig 1(a) shows the SEM images of the air-slot cavity realized by local line-defect-width modulation. Figure 1(b) shows the zoomed in SEM image of slot cavity highlighting the shifting of holes denoted by the black arrows (red: $5 \mathrm{~nm}$; green: $10 \mathrm{~nm}$; blue: 15 $\mathrm{nm})$. The red arrow in figure 1(a) highlights the propagation direction of injected input light through a channel waveguide which excites the slot cavity via direct tunneling coupling.

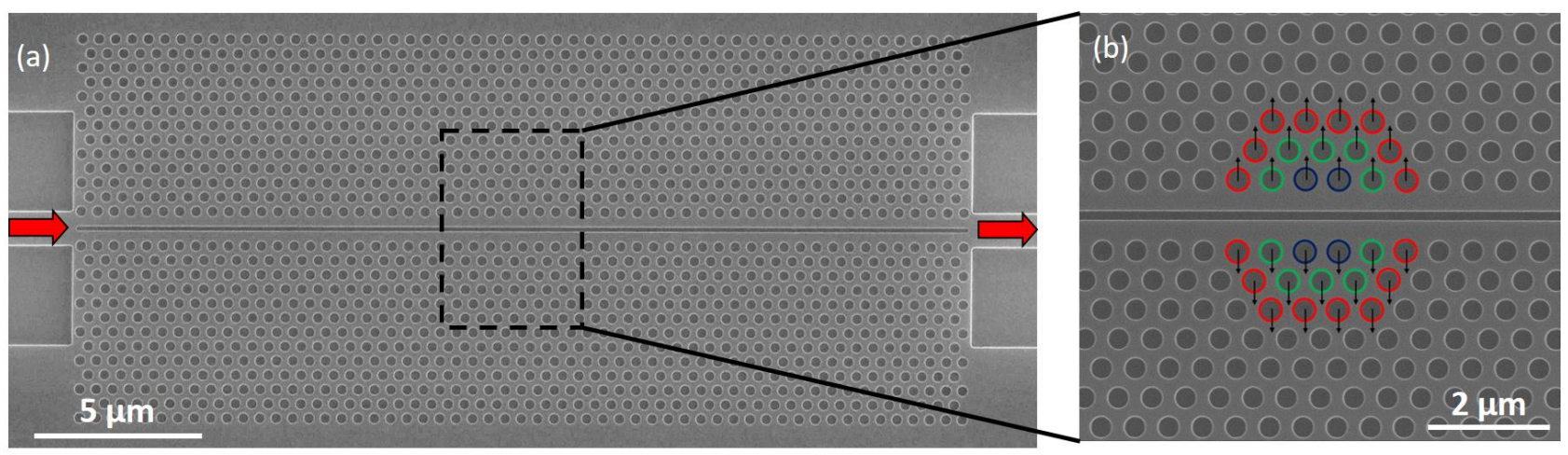

Figure 1 (a). SEM image of air-slot cavity, (b) zoomed in SEM image of slot cavity highlighting the shifting of holes denoted by the black arrows (red: $5 \mathrm{~nm}$; green: $10 \mathrm{~nm}$; blue: $15 \mathrm{~nm}$ ).

\section{EXPERIMENTAL CHARACTERIZATION OF AIR-SLOT CAVITY MODE}

In order to experimentally measure the Q-factor of the PhC slot cavity we have used an end-fire optical setup as shown in figure 2. This setup allows collection of the out-of-plane scattered light from the cavity surface. The photonic structure is excited with a TE-polarized, monochromatic diode laser. The out-of-plane scattered light from the excited cavity surface is collected through a high numerical aperture (0.95) microscope objective and detected with an infrared camera. The air-slot cavity is excited by direct tunneling scheme (in-line) as shown in Figure 1(a). 


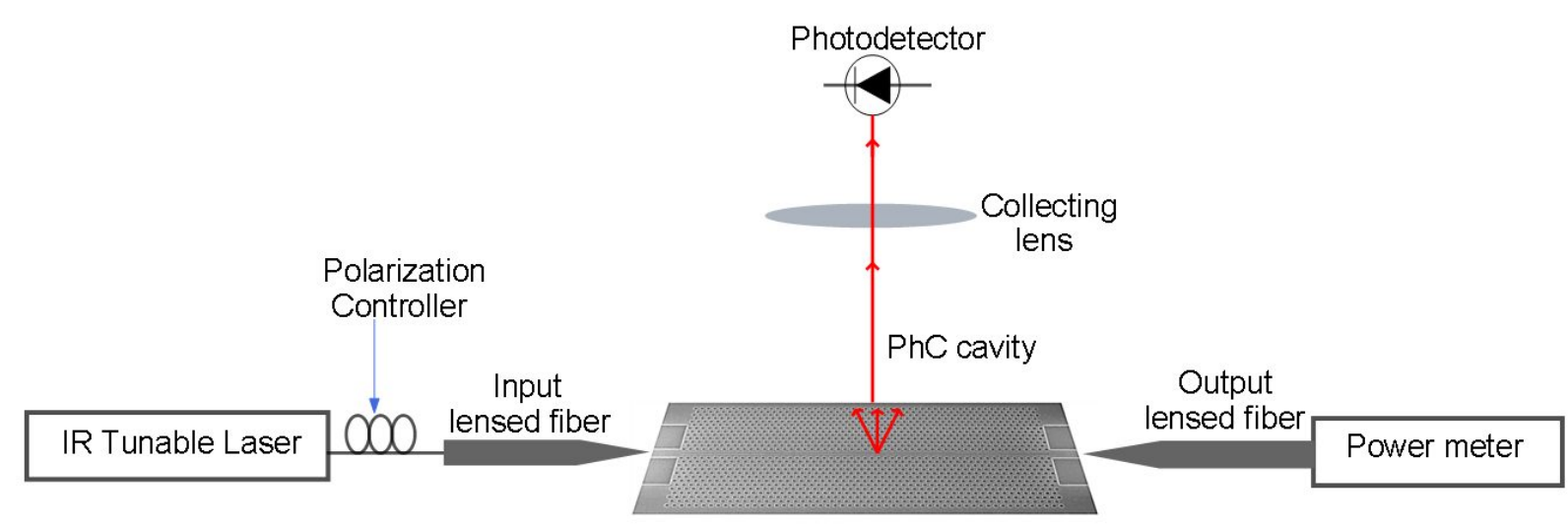

Figure 2. Schematic of the experimental apparatus used for characterize PhC cavity.

First, we investigated the cavities which was excited via direct tunneling. Figure 3(a) shows the transmission spectra of the in-line air-slot cavity membranes. The black curve in the figure is the measured intensity collected from the cavity surface and red curve is the Lorentzian fit of the transmission spectrum. Intensity pattern of the excited cavity at resonance wavelength is captured by an infrared camera is shown in Figure 3(b).

In order to probe the electric field distribution in the near field of a $\mathrm{PhC}$ slot cavity, we have mapped the mode of the inline air-slot cavity using a scattering near field optical technique. The mapping was done by a moving a dielectric nanotip close to near field of the slot cavity. When a dielectric nano-object approaches the near field of an optical cavity, the local refractive index changes. It results in a redshift of the wavelength of the cavity resonance and a simultaneous variation of the intensity stored in the cavity for an excitation at a single frequency. For weak perturbations, the intensity variation is proportional to the local cavity field intensity and to the volume of the nanoparticle. It follows that the spatially mapping the perturbation-induced intensity variation provides an image of the intensity distribution of the cavity field. The experimental knowledge of the distribution of the cavity field is important to predict the sensing capabilities of the cavity.

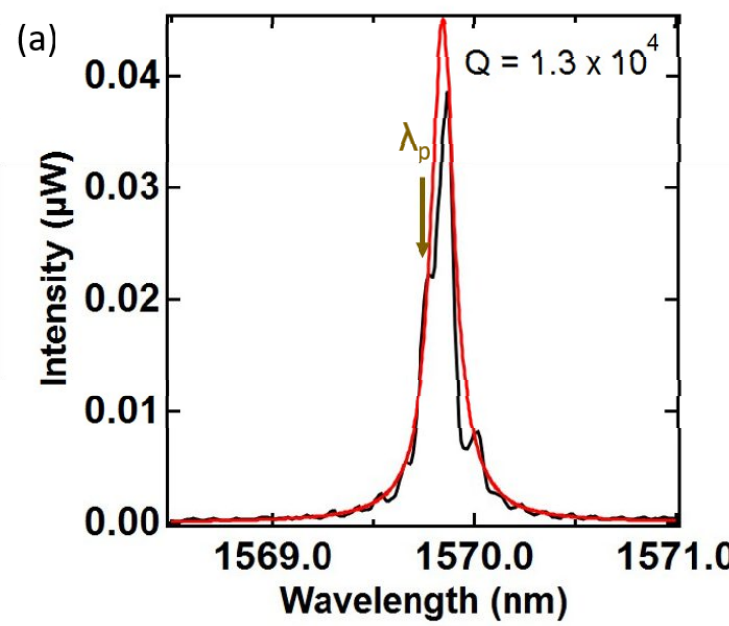

(b)

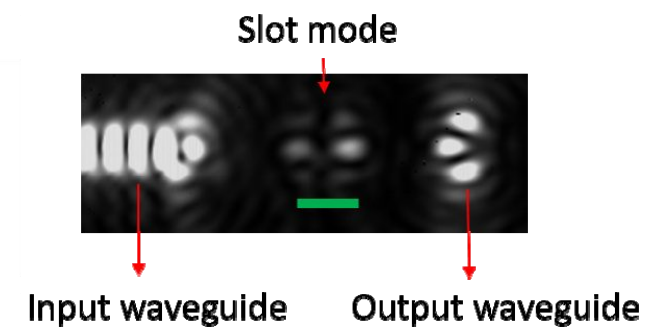

Figure. 3. (a) Transmission spectra of suspended PhC cavity (the blue arrow indicates the pump wavelength at which cavity was excited for mode mapping), (b) Intensity pattern of the cavity field at resonance wavelength (the green scale corresponds to 4 $\mu \mathrm{m})$.

We mount the silica fiber nano-tip on a piezoelectric stage and position it close to the backside of the cavity surface. The other end of the fiber nano-tip is connected to a $780 \mathrm{~nm}$ laser diode which helps in locating position of the nano-tip. This 
technique allows us to provide better understanding of impact of light coupling in PhC cavity modes and can also be very sensitive in distinguishing nanoparticle of various size.

We first measured the cavity resonance which is at $1569.7 \mathrm{~nm}$. While accurately keeping these alignments we blue shifted the pump wavelength $\left(\lambda_{\mathrm{p}}\right)$ by $0.15 \mathrm{~nm}$ (figure 3(a)), the tip was raster scanned in the near field of the cavity in $\mathrm{XY}$ plane from the backside while simultaneously recording the cavity transmittance (I). This measurement provides map of interaction between the fiber nano-tip and the confined cavity field allowed us to reconstruct the map of the cavity.

The interaction between the nano-tip fiber and the confined cavity field creates a map of the intensity variations of the scattered intensity from the cavity as shown in figure 4 . As it can be seen in the figure 4 , this map provides information about the maximum and minimum of electric field distribution inside the cavity. The scan step in $\mathrm{X}$ and $\mathrm{Y}$ direction is $100 \mathrm{~nm}$ and $10 \mathrm{~nm}$ respectively. The black color represents the minimum intensity whereas white color represents maximum intensity. Since mapping was at the pump wavelength which lies on the blue edge of the cavity spectrum and cavity resonance redshifts due to presence of the tip, the map should have minimum of intensity at the center. This does not seems be the case as shown in figure 4 . We believe that this discrepancy is due to the way cavity is excited and as in present case the barrier which excited the cavity via direct tunneling gets disturbed due to presence of the tip.

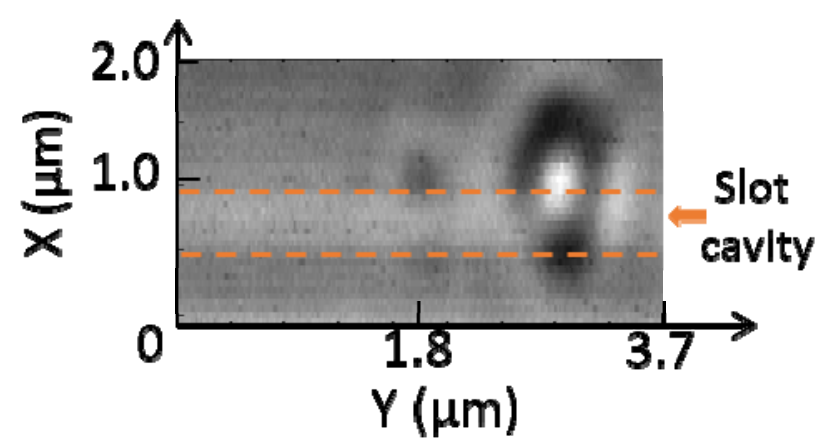

Figure. 4. Map of the intensity variation induced by the interaction between the nano-tip fiber and the air-slot cavity.

The movement of tip near the in-line slot cavity seemed to affect the light guiding in barrier of the cavity. As a result, it makes difficult to extract the intrinsic map of the cavity field. So, we opted to use side-coupled air-slot cavity in which case, cavity is excited by a side waveguide as shown in figure 5 .

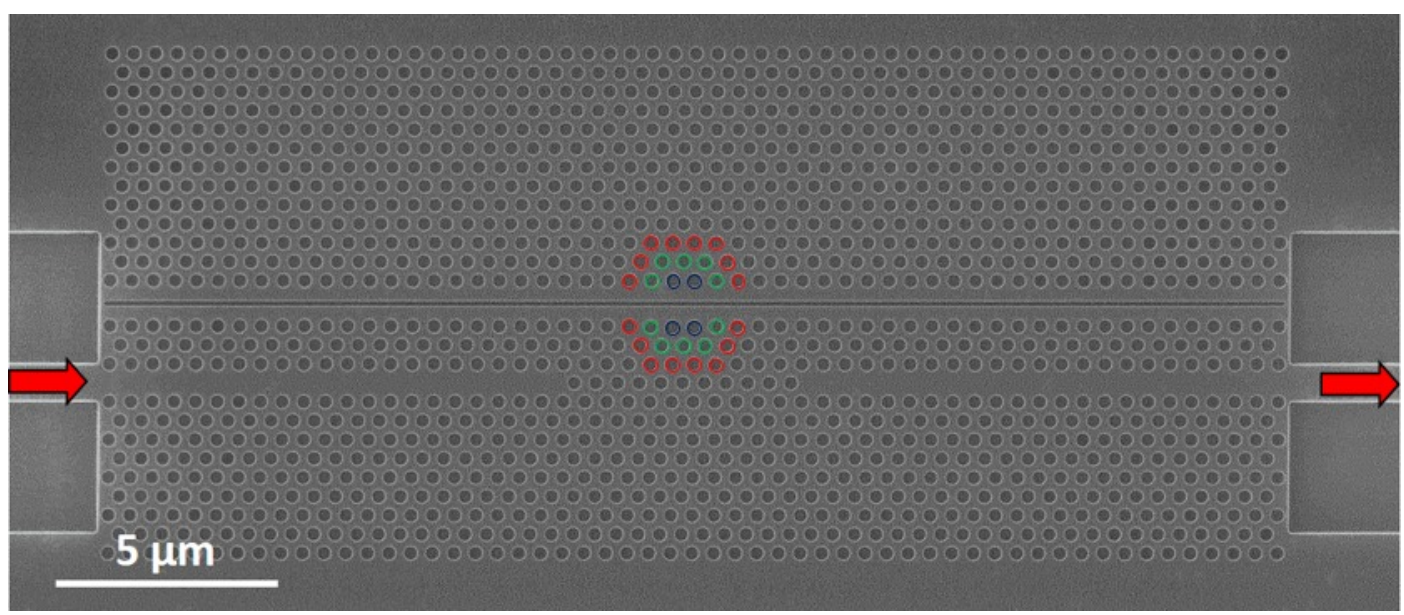

Figure 5. SEM image of side-coupled air-slot cavity.

In order to minimize the impact of the coupling, we designed and measured slot cavity with a side coupling configuration. No cavity mode observed in-side coupled slot cavities. Therefore, there is still a need to optimize the side 
coupling of such slot cavities. The investigation of air-slot cavities excited via different scheme will allow us to provide better understanding of field distribution inside the cavity.

\section{CONCLUSION}

We have investigated slot cavity mode with a scattering near field optical technique to probe the field distribution in the near field of a PhC slot cavity by scanning a nano-tip approaches the cavity surface. We show that how we control the positioning of a nano-tip near cavity surface and map any cavity mode. We have currently been able to probe slot cavity modes only in the case of an in-line coupling, which makes it difficult to disentangle the intensity variation due to the mode frequency shift from the ones resulting from the light coupling perturbation.

\section{REFERENCES}

[1] Y. Zhang, Y. Zhao, and Q. Wang, "Measurement of methane concentration with cryptophane e infiltrated photonic crystal microcavity," Sensors and Actuators B: Chemical, vol. 209, pp. 431-437, 2015.

[2] Y. Chang, Y.-Y. Jhu, and C.-J. Wu, "Temperature dependence of defect mode in a defective photonic crystal," Optics Communications, vol. 285, no. 6, pp. 1501-1504, 2012.

[3] T. Lu and P.-T. Lee, "Ultra-high sensitivity optical stress sensor based on double-layered photonic crystal microcavity," Optics express, vol. 17, no. 3, pp. 1518-1526, 2009.

[4] S. H. Mirsadeghi and J. F. Young, "Ultrasensitive diagnostic analysis of au nanoparticles optically trapped in silicon photonic circuits at sub-milliwatt powers," Nano letters, vol. 14, no. 9, pp. 5004-5009, 2014.

[5] Takayuki Yamamoto, Masaya Notomi, Hideaki Taniyama, Eiichi Kuramochi, Yutaka Yoshikawa, Yoshio Torii, and Takahiro Kuga, "Design of a high-Q air-slot cavity based on a width-modulated line-defect in a photonic crystal slab" Optics Express Vol. 16, Issue 18, pp. 13809-13817 (2008). 